eine gesunde Darmflora führt zu einer gesunden Scheidenflora und somit zu einem optimalen Schutz auch vor rezidivierenden Harnwegsinfekten, die uns in der gynäkologischen Praxis immer wieder begegnen und häufig den Einsatz von Antibiotika nach sich ziehen.

\section{Risiko für Sectio-Kinder}

Lactobazillen und Bifidobakterien werden ebenfalls in der Muttermilch nachgewiesen und auf den Säugling übertragen. Sie ermöglichen eine optimale Entwicklung der kindlichen Darmflora. Einfluss auf das kindliche Mikrobiom hat auch die Geburt: Bei einer Spontangeburt entwickelt sich in der Regel eine gute kindliche Darmflora. Kinder, die per Sectio entbunden werden und keinen Kontakt zur Scheidenflora hatten, weisen dagegen eine veränderte Kolonisation der Darmflora auf, die laut epidemiologischen Studien für eine Reihe von Erkrankungen verantwortlich gemacht werden kann. Demnach steigt das rela- tive Risiko für Asthma bronchiale, Allergien, Diabetes mellitus I und Adipositas um 19\% an. Als Gegenmaßnahme testete eine New Yorker Pilotstudie jüngst den sogenannten vaginalen Mikrobentransfer, um die Darmflora von Neugeborenen zu fördern und Mikroben von der Mutter auf ihr Kind zu übertragen. Dabei wurden die Kinder unmittelbar nach der Geburt mit einer Kompresse eingerieben, die vor Beginn des Kaiserschnitts in der Vagina der werdenden Mutter platziert wurde.

Bei der Behandlung der Dysbiose gewinnt der Einsatz von Probiotika und Präbiotika seit Jahren an Bedeutung. Probiotika sind lebende Organismen, die für den Aufbau einer gesunden Darmflora sorgen. Präbiotika sind pflanzliche Ballaststoffe, die eingesetzt werden, um nützliche Bakterien zu füttern und pathogene Bakterien auszuhungern.

Diagnostik und gegenenfalls die Therapie der Darmflora sowie das Screening der

\title{
Hygiene in der Praxis: ein Fall für Profis
}

$\mathrm{O}$ $\mathrm{b}$ angemeldet oder plötzlich und unerwartet im laufenden Praxisbetrieb: Das Infektionsschutzgesetz schränkt die Grundrechte des Praxisinhabers dahingehend ein, dass er Praxisbegehungen durch das Gesundheitsamt zu dulden hat. Sie erfolgen meist anlassbezogen, zum Beispiel aufgrund einer Patientenbeschwerde, und machen deutlich, dass die Hygiene in der Praxis schnell zur Stolperfalle geraten kann. Fündig werden die Kontrolleure meistens.

Während Nachbesserungen bei den allgemeinen Hygieneregeln, Dokumentationsverpflichtungen und Maßnahmen des Arbeitsschutzes etc. erfahrungsgemäß problemlos innerhalb der gesetzten Fristen umgesetzt werden können, sind strukturelle Veränderungen insbesondere beim ambulanten Operieren und nachzuweisender Sachkunde des Personals nicht auf die Schnelle aus dem Ärmel zu zaubern. Auch der Kurs „Hygienebeauftragter Arzt“ dauert seine Zeit und ist je nach Bundesland Pflicht, gegebenenfalls erst ab Ende 2016.
Bei klaren Verstößen gegen Hygienegrundlagen, etwa bei der Aufbereitung von Medizinprodukten, wird es richtig ernst: Ein immer noch in Betrieb befindlicher, antiker Heißluftsterilisator oder ähnliches kann durchaus zur Praxisschließung führen. In Anbetracht der behördlicherseits regelmäßig vorgetragenen Menge bestehender Mängel bei der Sterilgutaufbereitung sowohl im stationären als auch im ambulanten Bereich muss davon ausgegangen werden, dass hier vielerorts auch in Verbindung mit dem Qualitätsmanagement nach wie vor erheblicher Handlungsbedarf besteht.

Das gilt vor allem deshalb, weil im Falle eines wenn auch nur angenommenen Patientenschadens das Nicht-Einhalten von Vorschriften zur Beweislastumkehr führt. Selbst formale Verstöße gegen die komplexen Regeln des Sterilgut-Managements sind mit erheblichen Sanktionen belegt; straf- und steuerrechtliche Aspekte gilt es für Praxen, OP-Zentren, MVZ und Belegärzte im Einzelfall ebenfalls zu berücksichtigen.
Mundflora (Perio Safe Test) sind heute wichtige Bausteine zur Gesunderhaltung unserer Patientinnen. Sie erweitern das Spektrum der Präventionsmedizin, das wir im Rahmen einer innovativen Neuausrichtung gynäkologischer Praxen als Fachärzte für die Frau anbieten können.

Fortbildungen in Präventionsmedizin organisiert die GenoGyn seit acht Jahren. Die Mund- und Darmflora steht Mitte 2016 auf dem Programm, referiert von interntional bekannten Experten. Die Termine werden zeitnah unter www.genogyn.de veröffentlicht. Interessierte können sich bereits in der GenoGyn-Geschäftsstelle unter Telefon 0221 / 940505390 vormerken lassen.

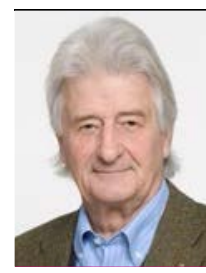

Dr. med. Jürgen Klinghammer Facharzt für Frauenheilkunde und Geburtshilfe Vorstand der GenoGyn

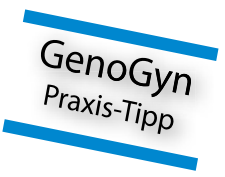

Fazit: Hygienemanagement in Eigenregie war gestern vertretbar, heute gehört es in die Hände von Profis, die aktuelle Normen und rechtliche Anforderungen individuell berücksichtigen und uns im besten Fall ein Komplettpaket "Sicheres Hygienemanagement" inklusive Sterilgutaufbereitung anbieten.

Entsprechende professionelle Lösungen hält unser Kooperationspartner Tietze \& Pozo Medizintechnik GmbH parat (www.tpm-alsdorf.de, Ansprechpartner: Prof. h.c. Dr. rer. oec. Manuel Pozo, Tel. 02404/94110) - für MitgliederPraxen der GenoGyn selbstverständlich zu Sonderkonditionen.

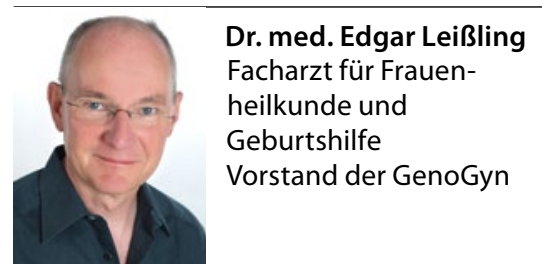

